\title{
The effects of the big storm events in the first half of 2015 on the radiation belts observed by EPT/PROBA-V
}

\author{
V. Pierrard ${ }^{1,2}$ and G. Lopez Rosson ${ }^{1}$ \\ ${ }^{1}$ Royal Belgian Institute for Space Aeronomy (IASB-BIRA), STCE and Space Physics, 3 av. Circulaire, \\ 1180 Brussels, Belgium \\ ${ }^{2}$ Université Catholique de Louvain (UCL), Center for Space Radiations (CSR) and Georges Lemaître Centre for Earth and \\ Climate Research (TECLIM), Earth and Life Institute (ELI), Place Louis Pasteur 3 bte L4.03.08, \\ 1348 Louvain-la-Neuve, Belgium
}

Correspondence to: V. Pierrard (viviane.pierrard@oma.be)

Received: 25 August 2015 - Revised: 15 December 2015 - Accepted: 16 December 2015 - Published: 21 January 2016

\begin{abstract}
With the energetic particle telescope (EPT) performing with direct electron and proton discrimination on board the ESA satellite PROBA-V, we analyze the highresolution measurements of the charged particle radiation environment at an altitude of $820 \mathrm{~km}$ for the year 2015 . On 17 March 2015, a big geomagnetic storm event injected unusual fluxes up to low radial distances in the radiation belts. EPT electron measurements show a deep dropout at $L>4$ starting during the main phase of the storm, associated to the penetration of high energy fluxes at $L<2$ completely filling the slot region. After 10 days, the formation of a new slot around $L=2.8$ for electrons of $500-600 \mathrm{keV}$ separates the outer belt from the belt extending at other longitudes than the South Atlantic Anomaly. Two other major events appeared in January and June 2015, again with injections of electrons in the inner belt, contrary to what was observed in 2013 and 2014. These observations open many perspectives to better understand the source and loss mechanisms, and particularly concerning the formation of three belts.
\end{abstract}

Keywords. Magnetospheric physics (Energetic particles trapped)

\section{Introduction}

Discovered by James Van Allen (Van Allen and Franck, 1959), the terrestrial radiation belts are toroidal regions surrounding Earth and filled with very energetic electrons and protons from hundreds of $\mathrm{keV}$ to hundreds of $\mathrm{MeV}$ trapped in the magnetic field of the Earth. The Earth's radiation belts consist of an inner belt lying within about 2 Earth radii $\left(R_{\mathrm{E}}\right)$ filled by energetic protons and electrons, and an outer belt lying between 3 and $7 R_{\mathrm{E}}$ dominated by energetic electrons. Between the inner and outer electron belts, there is a slot region where the electron flux is much lower as a result of precipitation by wave-particle interactions. While the inner radiation belt is fairly stable, the outer radiation belt is highly dynamic and the flux of relativistic $(\mathrm{MeV})$ electrons can change by several orders of magnitude on timescales from a few hours to a few days, increasing the risk to satellites (Pierrard and Benck, 2012). Observations of the outer electron radiation belt often show dropouts, i.e., sudden electron depletions during the main phase of storms, followed by injections occurring at lower radial distances. These changes in the radiation belt flux are driven by the interaction of the solar wind with the Earth's magnetosphere and can occasionally fill-in the slot region (Lemaire et al., 1996). The high energy electrons cause a range of problems for satellites like internal satellite charging effects while protons produce cumulative dose and damage, as well as prompt single event effects. Forecast is thus crucial and needs accurate measurements associated to good understanding of the physical mechanisms associated to the flux variations (Horne et al., 2013). Different empirical and/or physics-based models have been developed for electrons (for instance Vette, 1991; Brautigam et al., 1992) and for protons (Heynderickx et al., 1999).

Note that a third radiation belt was recently observed just after the launch of the two Van Allen Probes for energetic electrons (> 2 MeV), from 2 September 2013 and with a duration of around 4 weeks (Baker et al., 2013). This new ring 
Table 1. Energy ranges corresponding to each virtual channel of the EPT instrument for electrons and protons.

\begin{tabular}{lrr}
\hline $\begin{array}{l}\text { Energy } \\
\text { channels }\end{array}$ & $\begin{array}{r}\text { Electrons } \\
(\mathrm{MeV})\end{array}$ & $\begin{array}{r}\text { Protons } \\
(\mathrm{MeV})\end{array}$ \\
\hline 1 & $0.5-0.6$ & $9.5-13$ \\
2 & $0.6-0.7$ & $13-29$ \\
3 & $0.7-0.8$ & $29-61$ \\
4 & $0.8-1.0$ & $61-92$ \\
5 & $1-2.4$ & $92-126$ \\
6 & $2.4-8$ & $126-155$ \\
7 & $8-20$ & $155-182$ \\
8 & & $182-205$ \\
9 & & $205-227$ \\
10 & & $227-248$ \\
11 & & $>248$ \\
\hline
\end{tabular}

resulted from a combination of electron losses to the interplanetary medium and scattering by electromagnetic ion cyclotron waves to the Earth's atmosphere (Shprits et al., 2013).

Associated to recent observations of Van Allen Probe spacecraft, our results show that the radiation belts are sometimes different from the general feature. During the first half of 2015, several geomagnetic storms have modified the space radiations around the Earth, as described in the present work based on observations of the new performant EPT instrument that provides well discriminated flux measurements of electrons and protons.

\section{Data and analysis of the observations}

\subsection{The EPT instrument on PROBA-V}

Accurate measurements of energetic proton and electron fluxes without contamination are crucial to space weather predictions and to better understand the physical mechanisms implicated in the space radiation environment trapped in the magnetic field of the Earth. The EPT instrument (Cyamukungu et al., 2014) has especially been developed to obtain the best discrimination between the particle species and determine accurate particle flux measurements. The EPT was launched on 7 May 2013 to a LEO (low earth orbit) polar orbit at an altitude of $820 \mathrm{~km}$ onboard the ESA satellite PROBA-V with an inclination of $98.73^{\circ}$ and 10.30 a.m. as nominal local time at the descending node. The detector measures the particle fluxes for 7 virtual channels for electrons, 11 channels for protons and 11 channels for helium ions. Table 1 summarizes the different energy ranges corresponding to each virtual channel for electrons and protons used in the present study.

The characteristics of the instrument and the results of its first observations were presented and described in detail in Pierrard et al. (2014). In the present study, we report the formation of unexpected space radiation hazard appearing after a geomagnetic storm on 17 March 2015 and two other similar events that occurred respectively in January and June 2015. In Sect. 2, we show and analyze the electrons measurements, while protons are described in Sect. 3. Section 4 gives a discussion and conclusions on this new structure of the radiation belts.

\subsection{Characteristics of the 17 March 2015 event}

On 17 March 2015, the biggest geomagnetic storm observed by EPT since its launch in May 2013 reached a Dst index of $-223 \mathrm{nT}$. This event is also the biggest storm during the current solar cycle (up to now). The planetary geomagnetic index of Bartels reached $\mathrm{Kp}=7^{+}$. This event was associated with a sudden increase in solar wind density $n$, velocity $u$ and interplanetary magnetic field (IMF) intensity $B$ due to a shock created by a coronal mass ejection (CME). The event occurred because there were two flux increases, caused by two eruptions from the same active region. The first was a long duration C9.1 flare at 29W longitude location on the Sun with peak time at 15 March 01:25 UTC and accompanied by a partial-halo CME with a speed of $712 \mathrm{~km} \mathrm{~s}^{-1}$. The second increase follows an M1.2 flare (associated with a type II radio burst and a coronal dimming) at W32 location on 15 March with peak at 23:22 UTC. After the passage of the shock front, solar wind speed continued to increase reaching a peak of over $650 \mathrm{~km} \mathrm{~s}^{-1}$ around 10:45 UT on 17 March and a peak of the magnetic field over $35 \mathrm{nT}$ around 13:35 UT.

Figure 1 illustrates the different parameters of the solar wind (respectively IMF intensity $B$ in nT, $B_{z}$ (the $z$ component of IMF in $\mathrm{nT}$ ), density $n$ in $\mathrm{cm}^{-3}$, bulk velocity $u$ in $\mathrm{km} \mathrm{s}^{-1}$ ) and of geomagnetic activity (respectively $\mathrm{Kp}$ and Dst in nT) between 28 February and 11 April 2015. The $B_{z}$ peak went down to $-27 \mathrm{nT}$ during the CME.

The effect of this interplanetary shock caused a major event on geomagnetic activity and in the observations of the electron fluxes of the radiation belts. Figure 2 illustrates the electron fluxes observed by EPT/PROBA-V in Channel 1 (500-600 keV) as a function of the McIlwain (1961) parameter $L$ from 28 February until 13 April 2015. One can see the outer belt penetrating up to $L=2$ after the event, while the inner edge of the outer belt in this energy range is generally located at $L=3$ before the event. During previous lower geomagnetic events in 2013 and 2014, the outer belt generally did not penetrate at lower $L$ values than 2.7 (Pierrard et al., 2014). On the contrary, during the event of 17 March 2015, high fluxes are observed at radial distances as low as $L=2$, filling the usual slot region and reaching the inner belt that generally corresponds to the crossing of the South Atlantic Anomaly (SAA) at such low altitudes.

A few days after the event (around $23 \mathrm{March}$ ), the sector around $L=2.8$ starts to be gradually depleted for 500$600 \mathrm{keV}$ particles, so that a new slot region appears and grows in time. Due to this new depletion localized around $L=2.5$, the inner and outer belts are again separated, with 



Figure 1. Parameters of the solar wind measured by ACE (respectively IMF intensity $B$ in nT, $B_{z}$ (the $z$ component of IMF in nT), density $n$ in $\mathrm{cm}^{-3}$, bulk velocity $u$ in $\mathrm{km} \mathrm{s}^{-1}$ ) and geomagnetic activity indices (respectively Kp and Dst in nT) observed between 28 February and 11 April 2015. 


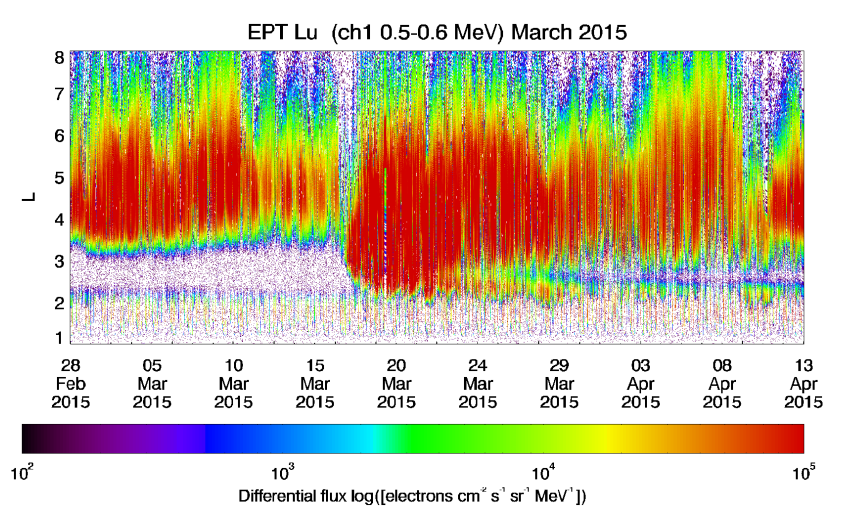

Figure 2. Electron fluxes observed by EPT/PROBA-V in Channel 1 (500-600 keV) as a function of $L$ between 28 February and 13 April 2015.

an upper edge of the inner belt highly more intense at $L=2.3$ than before the storm. The new electron population trapped at $L$ shells lower than 2.5 remains for more than a month, as its intensity decreases with time. The decay is most likely due to particle interactions with whistler mode hiss waves (e.g. Lyons et al., 1972; Lyons and Thorne, 1973; Meredith et al., 2007; Ripoll et al., 2014).

Note that at $L>4 R_{\mathrm{E}}$, the fluxes in the outer radiation belt drop out entirely during the main phase of the storm. Dropouts are commonly observed during storm events (Hudson et al., 1998).

As usual, the duration of the dropout is longer at high $L$ values. The fluxes remain low even after the storm has abated in the outer regions, which remain empty during more than 1 day.

Figure 3 illustrates the fluxes observed in the bin for $4.6<L<4.8,0.25<B<0.3 \mathrm{G}$ as an example of flux dropout around $L=4.7$. The electron flux drops by several orders of magnitude in just a few hours. After the depletion, the flux increases during the recovery phase and is larger a few days after the storm than before. Such a flux increase is generally observed after dropouts, but not always (Reeves et al., 2003). Sudden electron depletion observed during storm's main phase seems primarily a result of outward transport or an adiabatic change (Dst effect) rather than loss to the atmosphere (Turner et al., 2012).

Note that a similar behavior is also observed in higher channels of EPT.

Figure 4 illustrates the electron fluxes observed by EPT/PROBA-V in Channel $5(1-2.4 \mathrm{MeV})$ as a function of $L$ between 28 February and 13 April 2015. At these energies, high electron fluxes reach $L \sim 2.8$ but lower fluxes penetrate down to $L \sim 2.2$. The low flux level represents a new population that decays from 20 March to 1 April 2015, until the slot has been formed below $L<2.8$. But, referring to the prestorm period, between 28 February and 17 March, we can see the slot can be high up to $L \sim 3.4$ for these energies. Again,

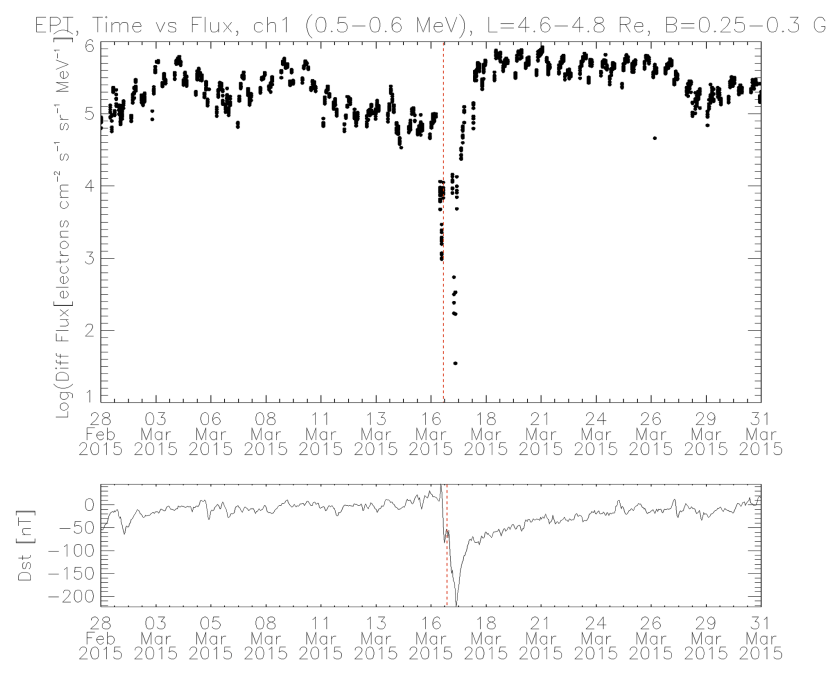

Figure 3. Electron fluxes observed by EPT/PROBA-V in Channel 1 $(500-600 \mathrm{keV})$ and in bin $4.6<L<4.8,0.25<B<0.3 \mathrm{G}$ as a function of time between 1 and 31 March 2015. A dropout is clearly visible during the main phase of the 17 March storm.



Figure 4. Same as Fig. 2 but for Channel 5 (1-2.4 MeV).

we are probably witnessing the scattering of $1-2 \mathrm{MeV}$ electrons by hiss waves, occurring between $L \sim 2.3$ and $L \sim 3.4$, as the combination of $(L, E)$ suggests, in agreement with theory (cf. Fig. 6 in Meredith et al., 2006; or Fig. 9 in Ripoll et al., 2014).

The slot position, as well as the belt position, is energy dependent.

Figure 5 shows the electron fluxes observed by EPT/PROBA-V in Channel $6(2.4-8 \mathrm{MeV})$ as a function of $L$ between 28 February and 13 April 2015. The fluxes penetrate a little bit deeper on 18 March than before the storm, but it is limited to $L=2.8$. This threshold was recently reported as an impenetrable barrier to ultra-relativistic electrons by Baker et al. (2014). The observation of no MeV electrons below $L=2.5$ (Figs. 4 and 5) in the inner belt is in agreement with the recent discovery from the Van Allen Probes of no 

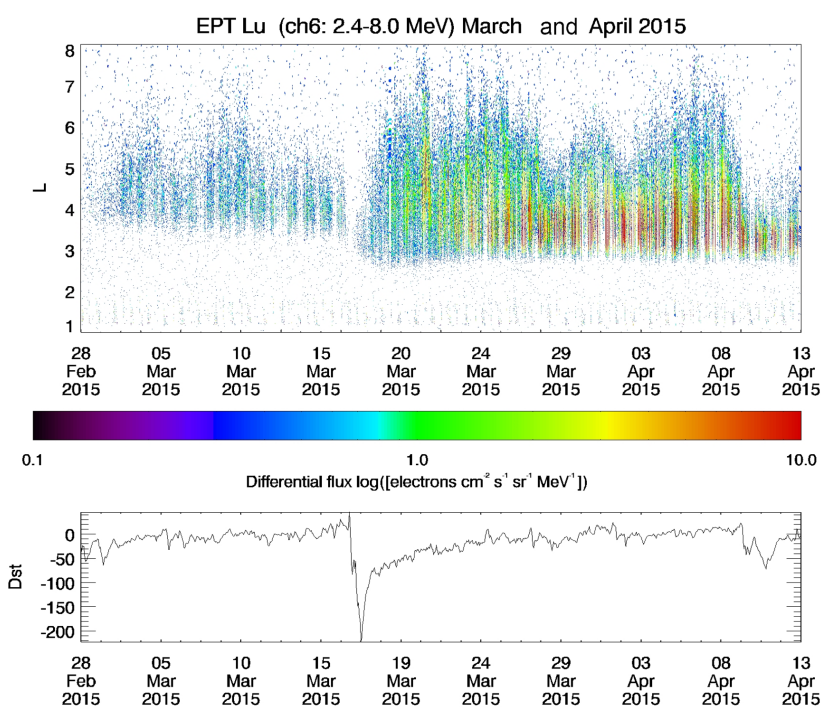

Figure 5. Electron fluxes observed by EPT/PROBA-V in Channel 6 $(2.4-8 \mathrm{MeV})$ as a function of $L$ between 28 February and 13 April 2015.

electrons (above threshold) of energy higher than $900 \mathrm{keV}$ in the inner belt (Fennell et al., 2015).

The two NASA satellites Van Allen Probes (VAP) A and $B$ also provide observations of the radiation belts at the same time. The orbits are quite different since VAP circulate close to the equatorial plane (inclination: $10^{\circ}$, Apoapsis: $5.8 R_{\mathrm{E}}$, Periapsis: $700 \mathrm{~km}$ ) while the orbit of PROBA-V is polar and LEO $(820 \mathrm{~km})$. A comparison with Van Allen Probe B/MAGEIS is illustrated in Fig. 6 (VAP A gives almost the same result). We use the MAGEIS data corrected from proton contamination that appeared mainly at $L<2$ in the inner belt (Claudepierre et al., 2014; Li et al., 2015). The resolution obtained with EPT is higher than with VAP because PROBA-V has a period of 101 min. and thus crosses the radiation belts more than 14 times per day while the orbital period of VAP is around $9 \mathrm{~h}$.

At the energy $742.5 \mathrm{keV}$, one can see the penetration of higher fluxes just above the inner belt. The formation of a third remnant belt is then visible: the first slot region remains at $L \sim 2$ because the penetration of the fluxes is a little bit higher than the outer edge of the inner belt, but a second slot is formed a few days later between $L \sim 2.3$ and $L \sim$ 2.9 , so that there is a short period with three belts and two slots (the new one being clearly deeper). Three belts have already been observed in the past (Cowen, 2013), even during longer periods, and especially by VAP just after the launch in September 2012 during 30 days (Baker et al., 2013).

Few electrons of energies from $600 \mathrm{keV}$ to $1 \mathrm{MeV}$ penetrate at such low radial distances of $L=2.3$ during the event of 17 March. Nevertheless, the few electrons that penetrate at these low L-shells have the same behavior as for Channel 1 of EPT: they are lost around $L=2.8$ in the slot region. The
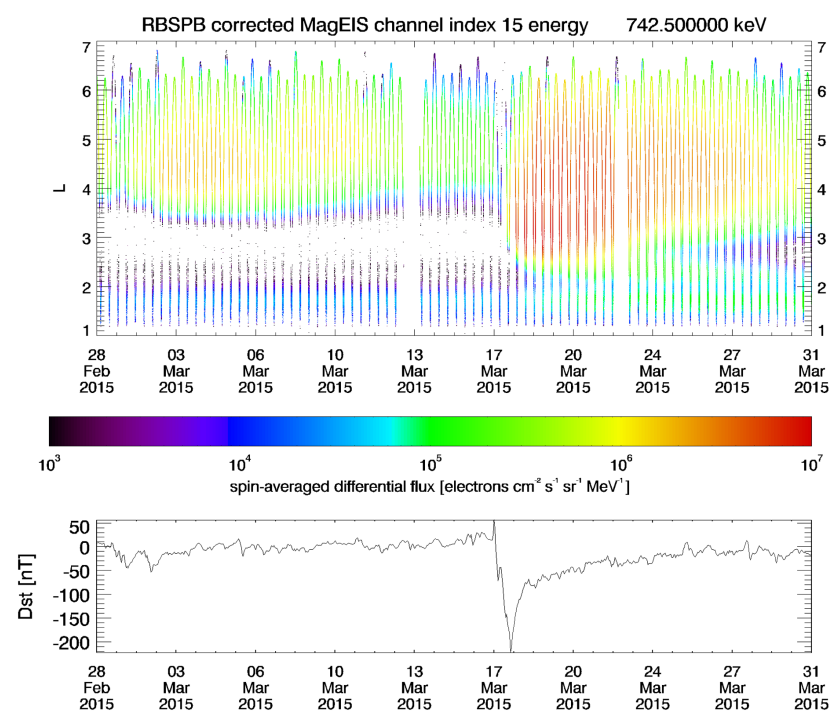

Figure 6. Corrected electron fluxes observed in Channel $742 \mathrm{keV}$ of Van Allen Probe/MAGEIS as a function of $L$ between 28 February and 31 March 2015.

others remain trapped and contribute to feed the inner belt during at least 1 month with a slightly decreasing flux at the outer edge of the inner belt.

\subsection{Analysis of spectra}

The electron spectra observed in two different $(L, B)$ bins are illustrated in Fig. 7 for 16 March, the day before the storm, for 17 March when the storm occurred and for 18 March, the day after the storm. The upper panel shows the bin $L=5.0$ $5.2, B=0.2-0.25$. We see clearly that the lowest fluxes were observed during the storm on 17 March (dropout), and that the highest fluxes are observed after the storm on 18 March. But for the 3 days, the spectra shape is the same so that the depletion and flux increase is similar for the different energy ranges. Bottom panel shows the bin $L=4.0-4.2, B=0.2-$ 0.25 . For this bin, 17 March has again the lowest fluxes, but the lowest energy channels $(<800 \mathrm{keV})$ are almost not depleted. The flux increase after the storm is on the contrary similar for all the different energy ranges. Studies of other storms confirm that the dropout mainly concerns the energetic electrons and mainly at high $L$. Note that the spectra show a nearly power-law shape instead of an exponential spectrum at high energies. The non-Maxwellian shape and the presence of different populations with different energies was already deduced earlier from AE8 electron spectra (Pierrard and Lemaire, 1996) in the radiation belts and even for protons with AP8 spectra (Pierrard and Borremans, 2012). The spectra vary with time so that the shape is different from one time to the other.

Flux variations observed by EPT as a function of Dst events will be used to complete the dynamic empirical TOP model of electron fluxes developed on the basis of averaged 

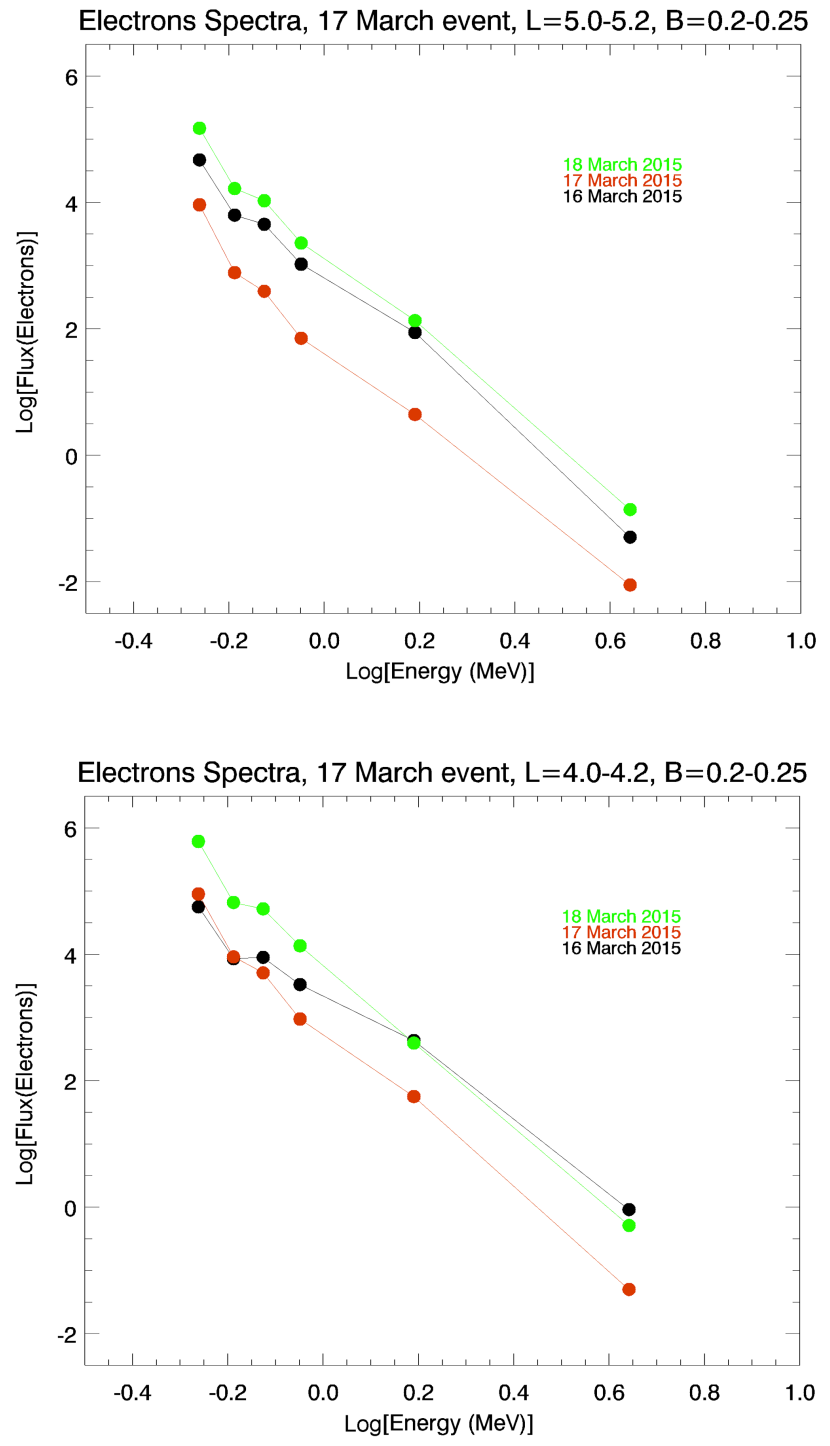

Figure 7. Spectra for two different $(L, B)$ bins observed by EPT on 16, 17 and 18 March 2015, corresponding respectively to the day before, during and after the storm. The upper panel shows the bin $L=5.0-5.2, B=0.2-0.25$. The lowest fluxes are observed during the storm, but for the 3 days, the spectra shape is the same. The bottom panel shows the bin $L=4.0-4.2, B=0.2-0.25$. For this bin, 17 March has also the lowest fluxes, but only the energies $>800 \mathrm{keV}$ show a dropout.

flux increase and decay times after storms observed in different ( $B, L$ bins) and for different energies by the LEO satellites SAC-C and DEMETER (Benck et al., 2010, 2013).

\subsection{Analysis of world maps}

World maps are also useful to illustrate LEO observations. Figure 8 shows the map of the electron fluxes observed in channel 1 by EPT/PROBA-V from 15 February until 15 March 2015 (before the event). While the inner belt cor-



Figure 8. Map of the electron fluxes observed by EPT/PROBA-V in Channel 1 between 15 February and 15 March 2015 (before the event).

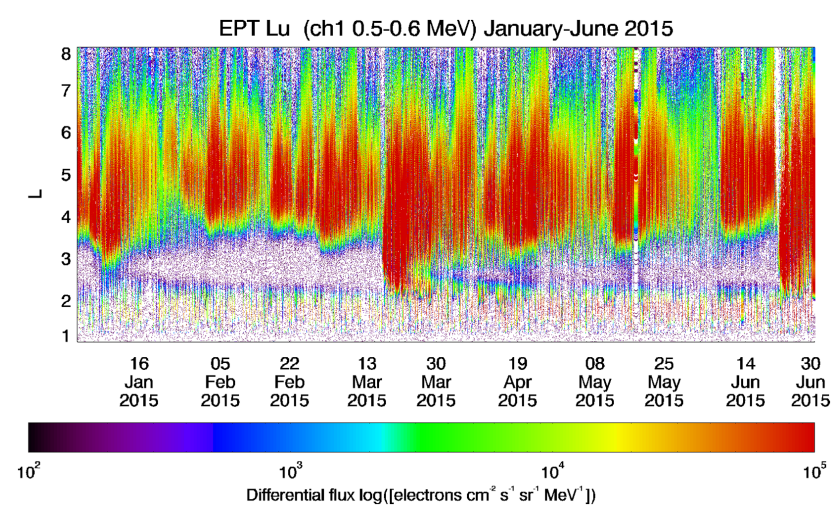

Figure 9. Electron fluxes observed by EPT/PROBA-V in Channel $1(500-600 \mathrm{keV})$ as a function of $L$ between 1 January and 30 June 2015.

responded only to the crossing of the SAA in 2013 and 2014 (see especially Pierrard et al., 2014), this is not anymore the case in February 2015: the presence of a thin belt appears at $L=2.3$ at all longitudes in the Northern and Southern Hemispheres at $820 \mathrm{~km}$ of altitude. This thin belt before the 17 March 2015 event is not visible in other channels.

It can be verified in Fig. 9 that this thin belt was created by another storm with a minimum Dst of $-99 \mathrm{nT}$ appearing on 7 January 2015. This storm injected some electrons of 500$600 \mathrm{keV}$ in the inner belt, and this belt was then visible at all longitudes at $820 \mathrm{~km}$ up to the event of March 2015 with a decreasing intensity. Note that another event on 23 June 2015 reached $-195 \mathrm{nT}$ and also injected electrons in the slot region.

Figure 10 shows the fluxes observed in Channel 1 from 16 March to 13 April 2015 after the mid-March storm: the 

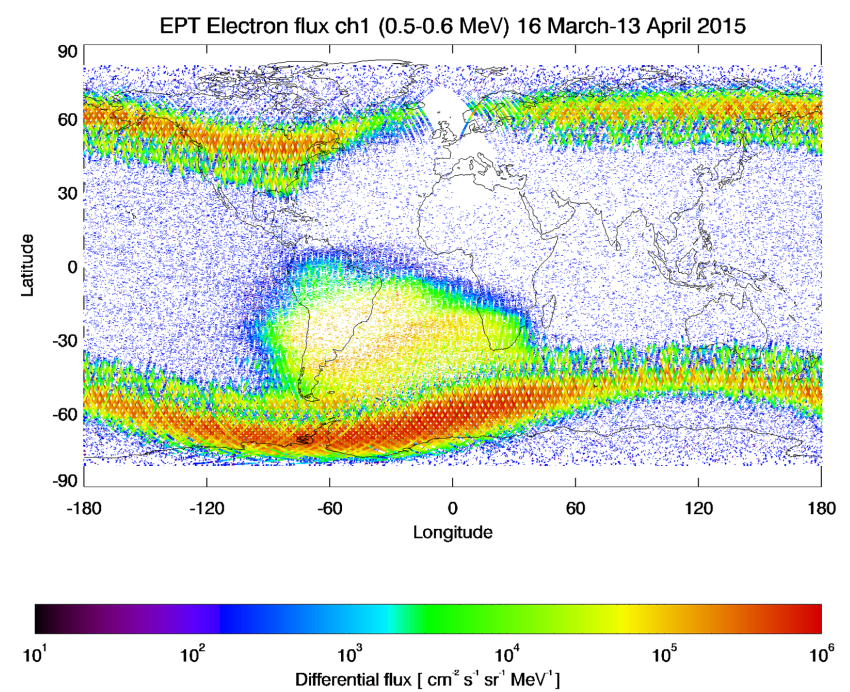

Figure 10. Map of the electron fluxes observed by EPT/PROBA-V in Channel 1 between 16 March and 13 April 2015 (after the event).
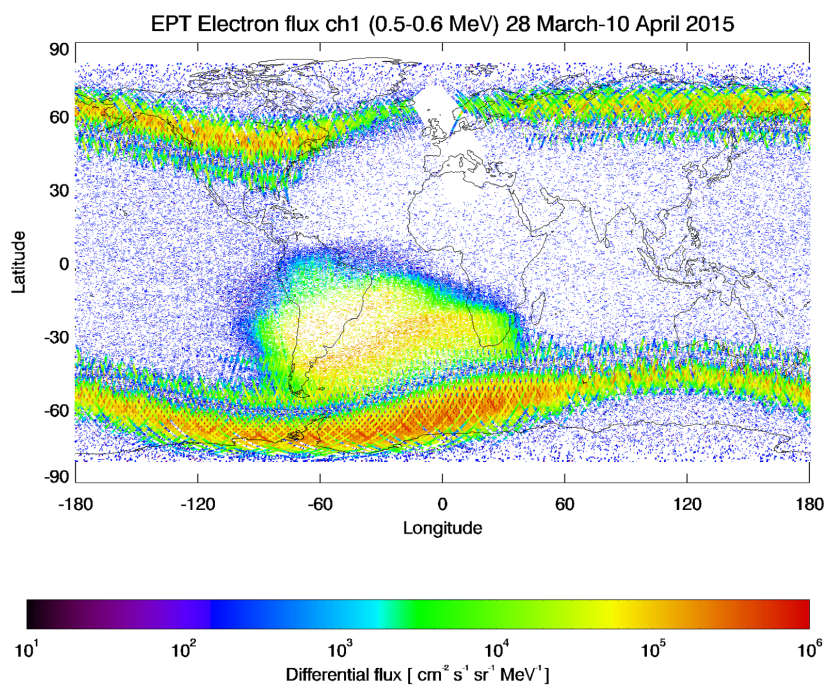

Figure 11. Map of the electron fluxes observed by EPT/PROBA-V in Channel 1 from 28 March up to 10 April 2015 (10 days after the event).

fluxes in the outer belt have highly increased and reached the inner belt. The fluxes are very high in the outer belt, much more than before the event (see Fig. 8) and they come closer to the SAA. The new slot appearing after 15 days is also clearly visible.

Figure 11 shows the same map but starting 10 days after the storm. The fluxes in the outer belt have been reduced and the new slot region is more visible. The new slot region, appearing here after typically 10 days, is believed to be a result of enhanced precipitation losses of energetic electrons due to their interactions with whistler waves in the magnetosphere (e.g. Lyons et al., 1972; Lyons and Thorne 1973;
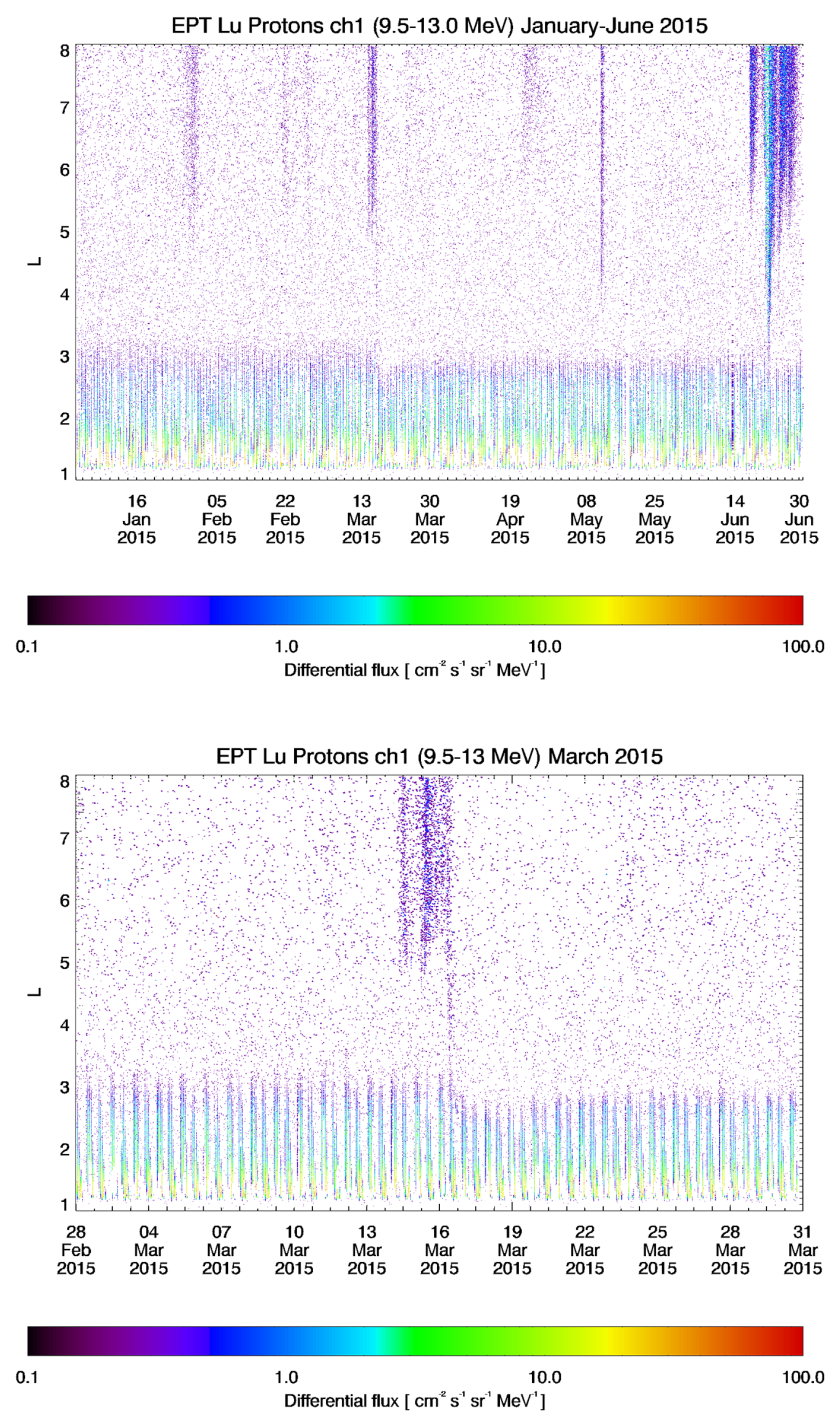

Figure 12. Proton fluxes observed by EPT/PROBA-V in Channel $1(9.5-13 \mathrm{MeV})$ as a function of $L$ between 1 January and 30 June 2015 (upper panel) and focused between 1 and 30 March 2015 (bottom panel) to emphasize that the March proton injection starts on 15 March 2015 due to the arrival of solar energetic protons (SEP event).

Glauert and Horne, 2005; Fung et al., 2006; Meredith et al., 2006; Sauvaud et al., 2008; Ripoll et al., 2014). Shprits et al. (2008a, b) have reviewed the different sources and loss mechanisms determining the dynamics of the outer belt for relativistic electrons.

\section{Protons}

Because EPT has a very good discrimination between the particle species, it is very interesting to investigate what happens to the protons during the same period of time. 


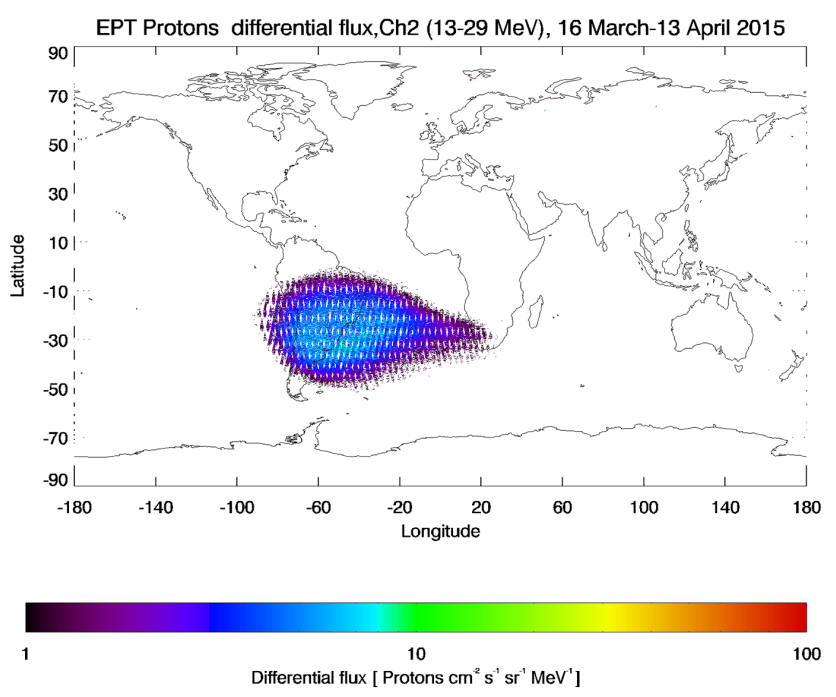

Figure 13. Map of the proton fluxes observed in Channel 2 by EPT/PROBA-V from 16 March until 13 April 2015.

Figure 12 shows the proton fluxes observed by EPT/PROBA-V in Channel $1(13-29 \mathrm{MeV})$ as a function of $L$ from 1 January up to 30 June 2015 . One can see protons suddenly injected and penetrating at high $\mathrm{L}$ values due to the event. This happened already on 15 March 2015 because the SEP event appeared before the geomagnetic storms associated to the CME. Sudden high-latitude proton fluxes appear also during the event of June where the injections were even stronger, longer in time and with higher fluxes. Such injections of energetic protons at high latitudes appear when there are SEP events (Pierrard et al., 2014). In June, three SEP events were observed on 18, 21 and 26 June 2015, and only these events of June inject also protons of higher energies visible in Channel 2. Note nevertheless that the injected proton fluxes are generally very low (see the color scale). The SEP event of 15 March 2015 reached a solar flux of 8 particles $\mathrm{cm}^{-2} \mathrm{~s}^{-1} \mathrm{sr}^{-1}$ for energies higher than $10 \mathrm{MeV}$, which is just below the threshold to be considered as a full SEP event.

In addition, we can see that after the event, the extent of the inner belt (corresponding to the crossing of the SAA at this low altitude of $820 \mathrm{~km}$ ) is slightly reduced, with an extension of the inner belt decreasing from $L=3$ to $L=2.8$. Similar injections are not observed at the higher energy channels (except for Channel 2 in June), neither for the helium ions.

Figure 13 shows the proton map observed by EPT/PROBA-V in Channel $2(9.5-13 \mathrm{MeV})$ between 16 March and 13 April 2015. The proton shape of the SAA is similar to the low energy electron SAA illustrated in Fig. 8, but a little less extended (for instance above the Pacific Ocean).

\section{Conclusions}

The event of 17 March 2015 is the highest geomagnetic storm that has been observed since the launch of PROBA-V in May 2013, with a Dst index as low as $-223 \mathrm{nT}$. We report here the injection of electrons in the inner belt after geomagnetic storms that make the inner belt visible at all longitude at $820 \mathrm{~km}$ and not anymore only in the SAA as in 2013 and 2014. The penetration of electrons from $500-600 \mathrm{keV}$ in the inner belt is followed by the formation of a well visible new slot region after around 10 days, helping us to understand the formation of three belt structures. Other major events appeared in January and June 2015, also with injection of electrons in the inner belt, while this was not observed during the 2 first years of observations. The good discrimination of the EPT instrument allows us also to observe the spectra to study the energy dependence of the electron flux variations during dropouts and after geomagnetic storms for instance, as well as simultaneous modifications in the proton observations. These new observations open many perspectives to better understand the source and loss mechanisms associated to the relativistic and non-relativistic particles in the radiation belts.

Acknowledgements. V. Pierrard and the Space Physics Department of IASB-BIRA thank the Belgian Science Policy - Space Research and Applications (Belspo) for the supplementary researcher program entitled "Scientific analysis of EPT (Energetic Particle Telescope) measurements". The research leading to these results has also received funding from the Scientific Federal Policy in the framework of the program Interuniversity Attraction Pole for the project P7/08 CHARM. The authors also thank K. Borremans and the team of Van Allen Probes for the comparison with VAP observations.

The development of the EPT instrument was realized with the support of the Belspo and of the European Space Agency (ESA) (contracts 20294/06/NL/JD (Phase A/B) and 22582/09/NL/AT (Phase $\mathrm{C} / \mathrm{D})$ ). The design of the EPT instrument and its integration on the PROBA-V satellite results from a fruitful Consortium between the Université Catholique de Louvain, the Royal Belgian Institute for Space Aeronomy and QinetiQ Space. The B.USOC and the control center in Redu provide data transmission and storage. The authors thank all the scientists who participated in the development of the EPT instrument, its integration on PROBA-V satellite and data validation. EPT data can be consulted on the website: http://csrsrv1.fynu.ucl.ac.be/csr_web/probav/index.html

The topical editor, G. Balasis, thanks J.-F. Ripoll and one anonymous referee for help in evaluating this paper. 


\section{References}

Baker, D. N., Kanekal, S. G., Hoxie, V. C., Henderson, M. G., Li, X., Spence, H. E., Elkington, S. R., Friedel, R. H. W., Goldstein, J., Hudson, M. K., Reeves, G. D., Thorne, R. M., Kletzing, C. A., and Claudepierre, S. G.: A long-lived relativistic electron storage ring embedded in Earth's outer Van Allen belt, Science, 340, 186-190, 2013.

Baker, D. N., Jaynes, A. N., Hoxie, V. C., Thorne, R. M., Foster, J. C., Li, X., Fennell, J. F., Wygant, J. R., Kanekal, S. G., Erickson, P. J., Kurth, W., Li, W., Ma, Q., Schiller, Q., Blum, L., Malaspina, D. M., Gerrard, A., and Lanzerotti, L. J.: An impenetrable barrier to ultrarelativistic electrons in the Van Allen radition belts, Nature Letter, 515, 531-534, doi:10.1038/nature13956, 2014.

Benck, S., Mazzino, L., Cyamukungu, M., Cabrera, J., and Pierrard, V.: Low altitude energetic electron lifetimes after enhanced magnetic activity as deduced from SAC-C and DEMETER data, Ann. Geophys., 28, 849-859, doi:10.5194/angeo-28-849-2010, 2010.

Benck, S., Cyamukungu, M., Cabrera, J., Mazzino, L., and Pierrard, V.: The Transient Observation-based Particle (TOP) model and its potential application in radiation effects evaluation, J. Space Weather Space Clim., 3, 1-10, doi:10.1051/SWSC/2013024, 2013.

Brautigam, D. S., Gussenhoven, M. S., and Mullen, E. G.: Quasistatic model of outer-zone electrons, IEEE Trans. Nucl. Sci., NS39-6, 1797, 1992.

Claudepierre, S. G., O’Brien, T. P., Blake, J. B., Fennell, J. F., Roeder, J. L., Clemmons, J. H., Looper, M. D., Mazur, J. E., Mulligan-Skov, T., Spence, H. E., Reeves, G. D., Friedel, R. H. W., Henderson, M. G., and Larsen, B. A.: A background correction algorithm for Van Allen Probes MagEIS electron flux measurements, J. Geophys. Res.-Space, 120, 5703-5727, doi:10.1002/2015ja021171, 2014.

Cowen, R.: Ephemeral third ring of radiation makes appearance around Earth, Nature News, doi:10.1038/nature.2013.12529, 2013.

Cyamukungu, M., Benck, S., Borisov, S., Bonnet, L., Grégoire, Gh., Cabrera, J., Desoete, B., Semaille, Ch., DeSaedeleer, J., Creve, G., VanRansbeek, E., Maes, J., Bonnewijn, S., Neefs, E., Pierrard, V., Lemaire, J., Moreau, D., Anciaux, M., Helderweirt, A., Litefti, K., Nieminen, P., Valtonen, E., Punkkinen, R., and Wojtek, H.: The Energetic Particle Telescope (EPT) on board Proba-V: description of a new science-class instrument for particle detection in space, IEEE Trans. Nuclear Sci., 61, 3667-3681, doi:10.1109/TNS.2014.2361955, 2014.

Fennell, J. F., Claudepierre, S. G., Blake, J. B., O’Brien, T. P., Clemmons, J. H., Baker, D. N., Spence, H. E., and Reeves, G. D.: Van Allen Pobes show the inner radiation zone contains no $\mathrm{MeV}$ electrons: ECT/MagEIS data, Geophys. Res. Lett., 42, 1283-1289, doi:10.1002/2014GL062874, 2015.

Fung, S. F., Shao, X., and Tan, L. C.: Long term variations of the electron slot region and global radiation belt structure, Geophys. Res. Let., 33, L04105, doi:10.1029/2005GL024891, 2006.

Glauert, S. A. and Horne, R. B.: Calculation of pitch angle and energy diffusion coefficients with the PADIE code, J. Geophys. Res., 110, A04206, doi:10.1029/2004JA010851, 2005.

Heynderickx, D., Kruglanski, M., Pierrard, V., Lemaire, J., Looper, M. D., and Blake, J. B.: A low altitude trapped proton model for solar minimum conditions based on SAMPEX/PET data, IEEE Trans. Nuclear Sci., 46, 1475-1480, 1999.
Horne R. B., Glauert, S. A., Meredith, N. P., Boscher, D., Maget, V., Heynderickx, D., and Pitchford, D.: Space weather impacts on satellites and forecasting the Earth's electron radiation belts with SPACECAST, Space Weather, 11, 169-186, 2013.

Hudson, M. K., Marchenko, V. A., Roth, I., Temerin, M., Blake, J. B., and Gussenhoven, M. S.: Radiation belt formation during storm sudden commencements and loss during main phase, Adv. Space Res., 214, 597-607, 1998.

Lemaire, J. F., Heynderickx, D., and Baker, D. N. (Eds.): Radiation Belts, Models and Standards, Geophysical Monograph, AGU, 97, 1996.

Li, X., Selesnick, R. S., Baker, D. N., Jaynes, A. N., Kanekal, S. G., Schiller, Q., Blum, L., Fennell, J., and Blake, J. B.: Upper limit on the inner radiation belt $\mathrm{MeV}$ electron intensity, J. Geophys. Res.-Space, 120, 1215-1228, doi:10.1002/2014JA020777, 2015.

Lyons, L. R. and Thorne, R. M.: Equilibrium structure of radiation belt electrons, J. Geophys. Res., 78, 2142-2149, doi:10.1029/JA078i013p02142, 1973.

Lyons, L. R., Thorne, R. M., and Kennel, C. F.: Pitch-angle diffusion of radiation belt electrons within the plasmasphere, J. Geophys. Res., 77, 3455-3474, doi:10.1029/JA077i019p03455, 1972.

McIlwain, C. E.: Coordinates for Mapping the Distribution of Magnetically Trapped Particles, J. Geophys. Res., 66, 3681-3691, 1961.

Meredith, N. P., Horne, R. B., Glauert, S. A., and Anderson, R. R.: Slot region electron loss timescales due to plasmaspheric hiss and lightning-generated whistlers, J. Geophys. Res., 112, A08214, doi:10.1029/2007JA012413, 2007.

Pierrard, V. and Benck, S.: The Dynamics Of The Terrestrial Radiation Belts And Its Links To The Plasmasphere, in Space weather: the space environment, AIP Conf. Proc., 1500, 216, doi:10.1063/1.4768769, 2012.

Pierrard, V. and Borremans, K.: Fitting the AP8 spectra to determine the proton momentum distribution functions in space radiations, Radiat. Meas., 47, 401-405, doi:10.1016/j.radmeas.2012.04.002, 2012.

Pierrard, V. and Lemaire, J.: Fitting the AE-8 energy spectra with two maxwellian functions, Radiat. Meas., 26, 333-337, 1996.

Pierrard, V., Lopez Rosson, G., Borremans, K., Lemaire, J., Maes, J., Bonnewijn, S., Van Ransbeeck, E., Neefs, E., Cyamukungu, M., Benck, S., Bonnet, L., Borisov, S., Cabrera, J., Grégoire, G., Semaille, C., Creve, G., De Saedeleer, J., Desoete, B., Preud'homme, F., Anciaux, M., Helderweirt, A., Litefti, K., Brun, N., Pauwels, D., Quevrin, C., Moreau, D., Punkkinen, R., Valtonen, E., Wojtek, H., Nieminen, P.: The Energetic Particle Telescope: First results, Space Science Rev., 184, 87-106, doi:10.1007/s11214-014-0097-8, 2014.

Reeves, G. D., McAdams, K. L., Friedel, R. H. W., and O'Brien, T. P.: Acceleration and loss of relativistic electrons during geomagnetic storms, Geophys. Res. Lett., 30, 36-1-36-4, doi:10.1029/2002GL016513, 2003.

Ripoll, J.-F., Chen, Y., Fennell, J. F., and Friedel, R. H. W.: On long decays of electrons in the vicinity of the slot region observed by HEO3, J. Geophys. Res.-Space, 119, 460-478, doi:10.1002/2014JA020449, 2014.

Sauvaud, J.-A., Maggiolo, R., Jacquey, C., Parrot, M., Berthelier, J.J., Gamble, R. J., and Rodger, C. J.: Radiation belt electron pre- 
cipitation due to VLF transmitters: Satellite observations, Geophys. Res. Lett., 35, L09101,doi:10.1029/2008GL033194, 2008.

Shprits, Y. Y., Elkington, S. R., Meredith, N. P., and Subbotin, D. A.: Review of modeling of losses and sources of relativistic electrons in the outer belt I: Radial transport, J. Atmos. Sol. Terr. Phys., 70, 1679-1693, doi:10.1016/j.jastp.2008.06.008, 2008a.

Shprits, Y. Y., Elkington, S. R., Meredith, N. P., and Subbotin, D. A.: Review of modeling of losses and sources of relativistic electrons in the outer belt II: Local acceleration and loss, J. Atmos. Sol. Terr. Phys., 70, 1694-1713, doi:10.1016/j.jastp.2008.06.014, 2008b.

Shprits, Y. Y., Subbotin, D., Drozdov, A., Usanova, M. E., Kellerman, A., Orlova, K., Baker, D. N., Turner, D. L., and Kim, K.C.: Unusual stable trapping of the ultrarelativistic electrons in the Van Allen radiation belts, Nature Phys., 9, 699-703, 2013.
Turner, D. L., Shprits, Y., Hartinger, M., and Angelopoulos, V.: Explaining sudden losses of outer radiation belt electrons during geomagnetic storms, Nature Phys., 8, 208-212, doi:10.1038/nphys2185, 2012.

Van Allen, J. A. and Frank, L. A.: Radiation Measurements to 658300 Kilometers with Pioneer IV, Nature, 184, 219-224, 1959.

Vette, J. I.: The AE-8 Trapped Electron Model Environment, NSSDC/WDC-A-R\&S 91-24, 1991. 